\title{
Reciprocity and mutual impedance formulas within lossy cavities
}

\author{
F. Gronwald and E. Blume
}

Institute for Fundamental Electrical Engineering and EMC, Otto-von-Guericke-University Magdeburg, Universitätsplatz 2, 39106 Magdeburg, Germany

\begin{abstract}
We discuss the validity of reciprocity and mutual impedance formulas within lossy cavities. Mutual impedance formulas are well-known from antenna theory and useful to describe the electromagnetic coupling between electromagnetic interference sources and victims. As an example the mutual impedance between two dipole antennas within a lossy rectangular cavity is calculated from a system of coupled Hallén's equations that efficiently is solved by the method of moments.
\end{abstract}

\section{Introduction}

An important framework to describe electromagnetic coupling between Electromagnetic Interference (EMI) sources and victims is antenna theory (, Lee, 1995; Tesche et al., 1997). Traditionally, antenna theory relies on electromagnetic field propagation in free space. It cannot directly be applied to an interior Electromagnetic Compatibility (EMC) problem that is limited by boundaries such that electromagnetic resonances are supported. Electromagnetic propagation in a resonating environment, like a cavity, for example, can considerably differ from that in free space. That is, if we wish to make use of a result of antenna theory in order to solve an interior EMC problem we have to check if it is valid not only in free space but also in a resonating environment.

An important example of this circumstance is provided by the integral formulas for mutual antenna impedances that are useful to model the mutual coupling between an EMI source and an EMI victim. These can be derived via the reciprocity theorem, and usually this is done under the assumption that transmitting and receiving antenna are located in free space (Elliott, 1981). In this paper we show that reciprocity and, thus, the integral mutual impedance formulas also are valid within lossy cavities.

The outline of the paper is as follows: Sect. 2 collects some standard formulas and notions in connection to reciprocity. Section 3 outlines the relation between reciprocity and surface boundary conditions while Sect. 4 links reciprocity to symmetry properties of the dyadic Green's function. Then,

Correspondence to: F. Gronwald

(frank.gronwald@et.uni-magdeburg.de) in Sect. 5, the mutual impedance formulas are introduced. We then review in Sect. 6 how to calculate antenna currents in a lossy rectangular cavity and compute, as an example, in Sect. 7 for a specific configuration of two antennas the mutual impedance. Sect. 8 provides some conclusions in view of EMC related issues.

\section{General remarks on reciprocity}

Suppose two sets of electric currents $\boldsymbol{J}^{a}$ and $\boldsymbol{J}^{b}$ exist in a region and produce electric and magnetic fields $\boldsymbol{E}^{a}, \boldsymbol{H}^{a}$ and $\boldsymbol{E}^{b}, \boldsymbol{H}^{b}$, respectively. Then, in the frequency domain, Maxwell's equations

$\nabla \times \boldsymbol{E}^{a, b}+j \omega \mu \boldsymbol{H}^{a, b}=\mathbf{0}$,

$\boldsymbol{\nabla} \times \boldsymbol{H}^{a, b}-j \omega \mu \boldsymbol{E}^{a, b}=\boldsymbol{J}^{a, b}$

are valid. We note that within Faraday's induction law Eq. (1) the magnetic field strength $\boldsymbol{B}$ already is replaced by the magnetic excitation $\boldsymbol{H}$ with the help of a linear constitutive equation of the form $\boldsymbol{B}=\mu \boldsymbol{H}$.

Dot multiplication of Eq. (1) with $\boldsymbol{H}^{b, a}$, and of Eq. (2) with $\boldsymbol{E}^{b, a}$ yields, after some elementary algebraic manipulations,

$\boldsymbol{\nabla} \cdot\left(\boldsymbol{E}^{b} \times \boldsymbol{H}^{a}-\boldsymbol{E}^{a} \times \boldsymbol{H}^{b}\right)=\boldsymbol{E}^{a} \cdot \boldsymbol{J}^{b}-\boldsymbol{E}^{b} \cdot \boldsymbol{J}^{a}$.

Integration of this local relation over a simply connected volume $V$ with surface $S$ that encloses all sources $\boldsymbol{J}^{a}, \boldsymbol{J}^{b}$ and application of Gauss's law leads to

$\int_{S}\left(\boldsymbol{E}^{b} \times \boldsymbol{H}^{a}-\boldsymbol{E}^{a} \times \boldsymbol{H}^{b}\right) \cdot d^{2} \boldsymbol{r}=$

$\int_{V}\left(\boldsymbol{E}^{a} \cdot \boldsymbol{J}^{b}-\boldsymbol{E}^{b} \cdot \boldsymbol{J}^{a}\right) d^{3} r$.

This relation often is called reciprocity theorem (Elliott, 1981). It is valid not only in free space but also in the presence of boundaries since it only is based on the local form of Maxwell's equations and the assumption of linear constitutive equations.

We define the reaction $\langle a, b>$ of field $a$ on source $b$ as the integral

$<a, b>:=\int_{V}\left(\boldsymbol{E}^{a} \cdot \boldsymbol{J}^{b}\right) d^{3} r$. 
Then reciprocity, in a less general sense than Eq. (4), is defined by the condition

$$
<a, b>=<b, a>\text {. }
$$

This relation proves to be particularly useful in antenna theory. Obviously, there are two possibilities to verify if reciprocity in this form is fulfilled. We can check if either the surface integral or the volume integral of Eq. (4) identically vanishes. In both cases we will need to know not only the dynamical Eqs. $(1,2)$ but also the boundary conditions imposed on the fields.

\section{Reciprocity and surface boundary conditions}

Let us concentrate on the surface integral of Eq. (4),

$I_{S}:=\int_{S}\left(\boldsymbol{E}^{b} \times \boldsymbol{H}^{a}-\boldsymbol{E}^{a} \times \boldsymbol{H}^{b}\right) \cdot d^{2} \boldsymbol{r}$.

We now show that $I_{S}$ vanishes both in free space and inside a lossy cavity.

1. Free Space: This is the standard case. If the sources of the fields are located in free space the surface $S$ can be chosen as the surface of a sphere with radius $r$ that tends to infinity, $r \rightarrow \infty$. Then the appropriate surface boundary condition on the fields is given by the radiation condition. It ensures that the integrand $\left(\boldsymbol{E}^{b} \times \boldsymbol{H}^{a}-\boldsymbol{E}^{a} \times \boldsymbol{H}^{b}\right)$ falls off faster than $1 / r^{2}$ (Van Bladel, 1985). It follows that the surface integral $I_{S}$ vanishes and reciprocity in the form Eq. (6) is ensured.

2. Inside a lossy cavity: If $S$ contains material boundaries we need appropriate boundary conditions that relate on $S$ the electric field strength $\boldsymbol{E}$ to the magnetic excitation $\boldsymbol{H}$. A fairly general first order boundary condition has been given by Leontovich, see Senior and Volakis (1995), for example,

$\boldsymbol{n} \times \boldsymbol{E}=Z \boldsymbol{n} \times(\boldsymbol{n} \times \boldsymbol{H})$,

with $\boldsymbol{n}$ the outward unit vector normal to $S$ and $Z$ the intrinsic impedance of the boundary. We then have

$$
\begin{aligned}
& \left(\boldsymbol{E}^{b} \times \boldsymbol{H}^{a}\right) \cdot \boldsymbol{n}=\left(\boldsymbol{n} \times \boldsymbol{E}^{b}\right) \cdot \boldsymbol{H}^{a} \\
& =Z\left(\boldsymbol{n} \times\left(\boldsymbol{n} \times \boldsymbol{H}^{b}\right)\right) \cdot \boldsymbol{H}^{a} \\
& =Z\left[\left(\boldsymbol{n} \cdot \boldsymbol{H}^{a}\right)\left(\boldsymbol{n} \cdot \boldsymbol{H}^{b}\right)+\boldsymbol{H}^{a} \cdot \boldsymbol{H}^{b}\right] .
\end{aligned}
$$

Clearly, this is invariant under exchange of $a$ and $b$. Since $d^{2} \boldsymbol{r}=\boldsymbol{n} d^{2} r$ it follows from Eq. (7) that the surface integral vanishes. Therefore the Leontovich boundary condition leads to reciprocity in the form Eq. (6).

Combinations of these two cases are also conceivable. For example, a cavity with openings can also be considered as long as the volume $V$ is simply connected such that we may apply Gauss's theorem to arrive at the integral relationship Eq. (4).

\section{Reciprocity as expressed by dyadic Green's functions}

To verify reciprocity we can also inspect the volume integral

$$
\begin{aligned}
& I_{V}:=\int\left(\boldsymbol{E}^{a} \cdot \boldsymbol{J}^{b}-\boldsymbol{E}^{b} \cdot \boldsymbol{J}^{a}\right) d^{3} r \\
& =<a, b>-<b, a>
\end{aligned}
$$

in order to see under which conditions it vanishes. While in the previous section the fields $\boldsymbol{E}$ and $\boldsymbol{H}$ got related by a surface boundary condition the field $\boldsymbol{E}$ and electric current $\boldsymbol{J}$ are related by a dyadic Green's function $\overline{\overline{\boldsymbol{G}}}^{E}$ if linearity is assumed,

$\boldsymbol{E}(\boldsymbol{r})=\int \overline{\overline{\boldsymbol{G}}}^{E}\left(\boldsymbol{r}, \boldsymbol{r}^{\prime}\right) \boldsymbol{J}\left(\boldsymbol{r}^{\prime}\right) d^{3} r^{\prime}$.

One should note that the Green's function $\overline{\overline{\boldsymbol{G}}}^{E}$ already contains the solution of Maxwell's equations with respect to specified boundary conditions.

We express the currents $\boldsymbol{J}^{a, b}$ by the motion of elementary charges $q_{i}$ at positions $\boldsymbol{r}_{i}^{a, b}=\boldsymbol{r}_{i}^{a, b}(t)$ that move with velocities $v_{i}^{a, b}$,

$\boldsymbol{J}^{a, b}(\boldsymbol{r})=\sum_{i} q_{i} \boldsymbol{v}_{i}^{a, b} \delta\left(\boldsymbol{r}-\boldsymbol{r}_{i}^{a, b}\right)$.

Then it follows from Eq. (14) that the electric fields $\boldsymbol{E}^{a, b}$ are given by

$\boldsymbol{E}^{a, b}(\boldsymbol{r})=\sum_{i} \overline{\overline{\boldsymbol{G}}}^{E}\left(\boldsymbol{r}, \boldsymbol{r}_{i}^{a, b}\right) q_{i} \boldsymbol{v}_{i}^{a, b}$.

With these expansions we find

$$
\begin{aligned}
& \int\left(\boldsymbol{E}^{a} \cdot \boldsymbol{J}^{b}-\boldsymbol{E}^{b} \cdot \boldsymbol{J}^{a}\right) d^{3} r \\
& =\int\left(\sum_{i}\left(\overline{\overline{\boldsymbol{G}}}^{E}\left(\boldsymbol{r}, \boldsymbol{r}_{i}^{a}\right) q_{i} \boldsymbol{v}_{i}^{a}\right) \cdot \sum_{j}\left(q_{j} \boldsymbol{v}_{j}^{b} \delta\left(\boldsymbol{r}-\boldsymbol{r}_{j}^{b}\right)\right)\right. \\
& \left.-\sum_{j}\left(\overline{\overline{\boldsymbol{G}}}^{E}\left(\boldsymbol{r}, \boldsymbol{r}_{j}^{b}\right) q_{j} \boldsymbol{v}_{j}^{b}\right) \cdot \sum_{i}\left(q_{i} \boldsymbol{v}_{i}^{a} \delta\left(\boldsymbol{r}-\boldsymbol{r}_{i}^{a}\right)\right)\right) d^{3} r \\
& =q_{i} q_{j} \sum_{i, j}\left(\left(\overline{\bar{G}}^{E}\left(\boldsymbol{r}_{j}^{b}, \boldsymbol{r}_{i}^{a}\right) \boldsymbol{v}_{i}^{a}\right) \cdot \boldsymbol{v}_{j}^{b}-\left(\overline{\overline{\boldsymbol{G}}}^{E}\left(\boldsymbol{r}_{i}^{a}, \boldsymbol{r}_{j}^{b}\right) \boldsymbol{v}_{j}^{b}\right) \cdot \boldsymbol{v}_{i}^{a}\right) .
\end{aligned}
$$

Therefore reciprocity, $<a, b>=<b, a>$, is obtained if

$G_{m n}^{E}\left(\boldsymbol{r}_{j}^{b}, \boldsymbol{r}_{i}^{a}\right)=G_{n m}^{E}\left(\boldsymbol{r}_{i}^{a}, \boldsymbol{r}_{j}^{b}\right)$

This symmetry property generally is fulfilled in the presence of perfectly conducting boundaries (Tai, 1994). It also holds in the case of lossy boundaries with boundary condition Eq. (8) if the non-vanishing impedance $Z$, in the framework of perturbation theory, is accounted for by a complex wavenumber such that the form of the dyadic Green's function remains the same as in the lossless case. 


\section{Integral formulas for mutual impedance formulas}

We now turn to electromagnetic coupling between an EMI source and an EMI victim. In many cases this coupling is modeled by the mutual coupling between two linear antennas. Generally, if two antennas are excited by electromagnetic sources there will be voltages $V_{1}, V_{2}$ and currents $I_{1}, I_{2}$ at the antenna input terminals. These quantities are related to each other by a $2 \times 2$ impedance matrix (Elliott, 1981)

$Z=\left(\begin{array}{ll}Z_{11} & Z_{12} \\ Z_{21} & Z_{22}\end{array}\right)$

according to

$V_{1}=Z_{11} I_{1}+Z_{12} I_{2}$,

$V_{2}=Z_{21} I_{1}+Z_{22} I_{2}$,

compare Fig. 1. The impedance matrix is independent of the excitation and concisely characterizes the antenna configuration which is determined from the antenna geometries, the antenna positions and orientations, and their environment. The impedances $Z_{11}$ and $Z_{22}$ are the self impedances of antenna 1 and antenna 2 in the presence of antenna 2 and antenna 1 , respectively. The mutual impedances $Z_{12}$ and $Z_{21}$ are a measure for the electromagnetic coupling between both antennas. Even though impedance is a very basic notion one should keep in mind that it is only defined for sinusoidal time variation or, as a trivial case, for no time variation.

It is evident from Eqs. $(21,22)$ that the mutual impedances can be calculated as ratios between open-circuit voltages and input currents. A solution of the antenna boundary value problem immediately yields the input currents but does not yield the open-circuit voltages.

If the antennas are located in free space usual antenna theory provides formulas for the open-circuit voltages that are based on the reciprocity theorem and a thin-wire approximation. For the mutual impedance $Z_{12}$ this is the integral expression (Elliott, 1981)

$Z_{12}=-\frac{1}{I_{1}^{\mathrm{t}} I_{2}^{\mathrm{oc} 1}} \int_{\text {antenna } 2} \boldsymbol{E}^{\mathrm{t} 21}(\xi) \cdot \boldsymbol{J}_{2}^{\mathrm{oc} 1}(\xi) d \xi$.

The quantities in this expression refer to two different situations. The first is indexed by " $\mathrm{t}$ " and is given by a transmitting antenna 1 in absence of antenna 2 . That is, $I_{1}^{\mathrm{t}}$ is the input current of a transmitting antenna in absence of antenna 2 and $\boldsymbol{E}^{\mathrm{t} 21}(\xi)$ is the electric field at the position of antenna 2 which is generated by antenna 1 if antenna 2 is removed from its position. The second situation is indexed by "oc1" and is given by a transmitting antenna 2 in the presence of antenna 1 which is open-circuited. The corresponding quantities $I_{2}^{\mathrm{oc} 1}$ and $\boldsymbol{J}_{2}^{\mathrm{oc} 1}(\xi)$ refer to the input current and current distribution, respectively, of antenna 2 in this situation. Analogously,

$Z_{21}=-\frac{1}{I_{2}^{\mathrm{t}} I_{1}^{\mathrm{oc} 2}} \int_{\text {antenna } 1} \boldsymbol{E}^{\mathrm{t} 12}(\xi) \cdot \boldsymbol{J}_{1}^{\mathrm{oc} 2}(\xi) d \xi$

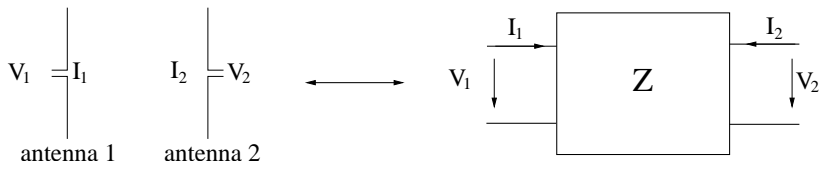

Fig. 1. Problem setup and its network equivalent.

Also the self impedances $Z_{11}$ and $Z_{22}$ can be calculated from similar formulas that are derived from the reciprocity principle. However, it is more convenient to excite, for the calculation of $Z_{11}$, antenna 1 by a fixed voltage source, say $V_{1}$, and then calculate the induced current $I_{1}^{\mathrm{oc} 2}$ at the input terminal of antenna 1 in the presence of the open-circuited antenna 2. This yields, according to Eq. (21),

$Z_{11}=\frac{V_{1}}{I_{1}^{\mathrm{oc} 2}}$.

In the same way we also obtain from Eq. (22)

$Z_{22}=\frac{V_{2}}{I_{2}^{\text {oc } 1}}$.

Equations $(25,26)$ can be used both in free space or within a cavity as long as the calculation of the currents $I_{1}^{\text {oc2 }}$ and $I_{2}^{\text {oc1 }}$ reflects the corresponding boundary conditions. In view of Eqs. $(23,24)$ it is important to note that these rely on reciprocity in the form Eq. (6) (Elliott, 1981). We have discussed in the previous sections that reciprocity also is valid in a lossy cavity if the boundary condition Eq. (8) is assumed. Therefore Eqs. $(23,24)$ can also be applied in this situation.

\section{Calculation of antenna currents within a cavity}

There are two standard integral equations that are in use to calculate the electric current on linear antennas. These are Hallén's equation and Pocklington's equation (Nakano, 1996). They are based on a vanishing tangential component of the magnetic vector potential $\boldsymbol{A}$ and the electric field $\boldsymbol{E}$, respectively, on the antenna surface which we assume to be perfectly conducting. If we split the total fields in a scattered and an incident part we can write Hallén's equation as

$$
\left[\int \overline{\bar{G}}^{A}\left(\boldsymbol{r}, \boldsymbol{r}^{\prime}\right) \boldsymbol{J}\left(\boldsymbol{r}^{\prime}\right) d \boldsymbol{r}^{\prime}+\boldsymbol{A}^{\mathrm{inc}}(\boldsymbol{r})\right]_{\tan }=\mathbf{0}
$$

and Pocklington's equation in the form

$$
\left[\int \overline{\bar{G}}^{E}\left(\boldsymbol{r}, \boldsymbol{r}^{\prime}\right) \boldsymbol{J}\left(\boldsymbol{r}^{\prime}\right) d \boldsymbol{r}^{\prime}+\boldsymbol{E}^{\mathrm{inc}}(\boldsymbol{r})\right]_{\tan }=\mathbf{0},
$$

with the dyadic Green's function $\overline{\overline{\boldsymbol{G}}}^{A}\left(\boldsymbol{r}, \boldsymbol{r}^{\prime}\right)$ for the magnetic vector potential and $\overline{\bar{G}}^{E}\left(\boldsymbol{r}, \boldsymbol{r}^{\prime}\right)$ the dyadic electric Green's function. Equations $(27,28)$ must be enforced on each antenna surface such that for $N$ antennas they constitute $N$ coupled integral equations.

Hallén's and Pocklington's equation are equivalent (Nakano, 1996), but which of both is more advantageous to 
use? The Coulomb singularity contained in $\overline{\overline{\boldsymbol{G}}}^{A}\left(\boldsymbol{r}, \boldsymbol{r}^{\prime}\right)$ is proportional to $1 /\left|\boldsymbol{r}-\boldsymbol{r}^{\prime}\right|$ and weaker as the Coulomb singularity of $\overline{\bar{G}}^{E}\left(\boldsymbol{r}, \boldsymbol{r}^{\prime}\right)$ which, in the source region, is proportional to $1 /\left|\boldsymbol{r}-\boldsymbol{r}^{\prime}\right|^{3}$. It follows that for numerical calculations Hallén's equation is preferable since it leads to algebraic equations that are numerically more stable. However, Hallén's equation has the disadvantage that it contains the incident field $\boldsymbol{A}^{\text {inc }}$. In practice, $\boldsymbol{E}^{\text {inc }}$ is provided by a given source rather than $\boldsymbol{A}^{\text {inc }}$. To obtain $\boldsymbol{A}^{\text {inc }}$ from $\boldsymbol{E}^{\text {inc }}$ one observes that, quite generally, in the Lorenz gauge

$\boldsymbol{E}=-\frac{j c}{k}\left[\nabla(\nabla \cdot \boldsymbol{A})+k^{2} \boldsymbol{A}\right]$.

Therefore a second order differential equation has to be solved to obtain $\boldsymbol{A}^{\text {inc }}$ from $\boldsymbol{E}^{\text {inc }}$ and this introduces two integration constants per antenna. These integration constants need to be determined from the vanishing of the antenna current at the end points of each antenna.

We prefer in the following numerical stability and focus on Hallén's equation. For a lossy, rectangular cavity we have calculated a representation of the Green's function $\overline{\bar{G}}^{A}\left(\boldsymbol{r}, \boldsymbol{r}^{\prime}\right)$ which is quickly convergent (Gronwald, 2003). This representation is based on the Ewald transformation (Ewald, 1921; Jordan et al., 1986; Park et al., 1998). In general, for a rectangular cavity and Cartesian coordinates $x, y$, and $z$, the dyad $\overline{\overline{\boldsymbol{G}}}^{A}\left(\boldsymbol{r}, \boldsymbol{r}^{\prime}\right)$ has three non-vanishing components,

$\overline{\overline{\boldsymbol{G}}}^{A}=G_{\mathrm{cav} x x}^{A} \hat{\mathbf{x}} \hat{\mathbf{x}}+G_{\mathrm{cav} y y}^{A} \hat{\mathbf{y}} \hat{\mathbf{y}}+G_{\mathrm{cav} z z}^{A} \hat{\mathbf{z}} \hat{\mathbf{z}}$.

The components $G_{\mathrm{cav} x x}^{A}, G_{\mathrm{cav} y y}^{A}$, and $G_{\mathrm{cav} z z}^{A}$ have the same explicit form and can be related to each other by cyclic exchange of $x, y$, and $z$. Hence, without loss of generality, it is sufficient to concentrate in the following on one component, say, $G_{\mathrm{cav} z z}^{A}$. The Ewald representation can be written in the combined form (Gronwald, 2003)

$G_{\mathrm{cav} z z}^{A}\left(\boldsymbol{r}, \boldsymbol{r}^{\prime}, k\right)=\underbrace{G_{\mathrm{cav} z z 1}^{A}\left(\boldsymbol{r}, \boldsymbol{r}^{\prime}, k\right)}_{\text {mode part }}+\underbrace{G_{\mathrm{cav} z z 2}^{A}\left(\boldsymbol{r}, \boldsymbol{r}^{\prime}, k\right)}_{\text {ray part }}$

with

$$
\begin{aligned}
& G_{\mathrm{cav} z z 1}^{A}=\frac{\mu}{8 l_{x} l_{y} l_{z}} \sum_{n_{x}, n_{y}, n_{z}=-\infty}^{\infty} \sum_{i=0}^{7} A_{i}^{z z} \times \\
& \frac{\exp \left(-\frac{k_{v}^{2}-k^{2}}{4 E^{2}}\right)}{k_{v}^{2}-k^{2}} \exp \left(j\left(k_{x} X_{i}+k_{y} Y_{i}+k_{z} Z_{i}\right)\right), \\
& G_{\mathrm{cav} z z 2}^{A}=\frac{\mu}{8 \pi} \sum_{m, n, p=-\infty}^{\infty} \sum_{i=0}^{7} A_{i}^{z z} \times \\
& {\left[\frac{\exp \left(j k R_{i, m n p}\right) \operatorname{erfc}\left(R_{i, m n p} E+j k / 2 E\right)}{R_{i, m n p}}\right.} \\
& \left.+\frac{\exp \left(-j k R_{i, m n p}\right) \operatorname{erfc}\left(R_{i, m n p} E-j k / 2 E\right)}{R_{i, m n p}}\right] .
\end{aligned}
$$

The variables $l_{x}, l_{y}$, and $l_{z}$ denote the dimensions of the rectangular cavity while the wave number $k_{v}$ is defined via

$k_{v}^{2}=k_{x}^{2}+k_{y}^{2}+k_{z}^{2}:=\left(\frac{n_{x} \pi}{l_{x}}\right)^{2}+\left(\frac{n_{y} \pi}{l_{y}}\right)^{2}+\left(\frac{n_{z} \pi}{l_{z}}\right)^{2}$

It corresponds to the resonances of the rectangular cavity with eigenfunctions

$\varphi_{\nu}(\boldsymbol{r}):=\sin \left(k_{x} x\right) \sin \left(k_{y} y\right) \cos \left(k_{z} z\right), \quad v \equiv n_{x} n_{y} n_{z}$.

Within the ray part the distances between original and mirrored sources to the observation point are given by,

$$
\begin{aligned}
& R_{i, m n p}\left(\boldsymbol{r}, \boldsymbol{r}^{\prime}\right)= \\
& \sqrt{\left(X_{i}+2 m l_{x}\right)^{2}+\left(Y_{i}+2 n l_{y}\right)^{2}+\left(Z_{i}+2 p l_{z}\right)^{2}}, \\
& X_{i}=\left\{\begin{array}{ll}
x-x^{\prime}, & i=0,1,2,3 \\
x+x^{\prime}, & i=4,5,6,7
\end{array},\right. \\
& Y_{i}=\left\{\begin{array}{ll}
y-y^{\prime}, & i=0,1,4,5 \\
y+y^{\prime}, & i=2,3,6,7
\end{array},\right. \\
& Z_{i}=\left\{\begin{array}{ll}
z-z^{\prime}, & i=0,2,4,6 \\
z+z^{\prime}, & i=1,3,5,7
\end{array} .\right.
\end{aligned}
$$

The correct sign rules of the mirror principle are taken into account by the factor

$A_{i}^{z z}=\left\{\begin{array}{ll}+1, & i=0,1,6,7 \\ -1, & i=2,3,4,5\end{array}\right.$.

Finally, "erfc" denotes the complementary error function and $E$ is an adjustable parameter which balances the contribution of mode and ray part. In average, the Ewald representation requires for convergence order of magnitudes less terms than the standard representations (Gronwald et al., 2002).

\section{Example}

Now we focus, for the sake of concreteness, on two dipole antennas within a rectangular cavity that are in parallel and aligned with the $z$-axis, compare Fig. 2 . If, for this configuration, a thin-wire approximation is applied we obtain from Hallén's equation (27) the coupled integral equations

$$
\begin{aligned}
& \int_{1} G_{\mathrm{cav} z z}^{A}\left(\boldsymbol{r}_{1}, \boldsymbol{r}_{1}^{\prime}\right) I_{1}\left(\boldsymbol{r}_{1}^{\prime}\right) d \boldsymbol{r}_{1}^{\prime}+\int_{2} G_{\mathrm{cav} z z}^{A}\left(\boldsymbol{r}_{1}, \boldsymbol{r}_{2}^{\prime}\right) I_{2}\left(\boldsymbol{r}_{2}^{\prime}\right) d \boldsymbol{r}_{2}^{\prime}= \\
& C_{11} \cos \left(k\left(z_{1}-z_{01}\right)\right)+C_{12} \sin \left(k\left(z_{1}-z_{01}\right)\right)+\frac{V_{01}}{2 j c} \sin \left(k\left|z_{1}-z_{01}\right|\right),
\end{aligned}
$$

$$
\begin{aligned}
& \int_{1} G_{\mathrm{cav} z z}^{A}\left(\boldsymbol{r}_{2}, \boldsymbol{r}_{1}^{\prime}\right) I_{1}\left(\boldsymbol{r}_{1}^{\prime}\right) d \boldsymbol{r}_{1}^{\prime}+\int_{2} G_{\mathrm{cav} z z}^{A}\left(\boldsymbol{r}_{2}, \boldsymbol{r}_{2}^{\prime}\right) I_{2}\left(\boldsymbol{r}_{2}^{\prime}\right) d \boldsymbol{r}_{2}^{\prime}= \\
& C_{21} \cos \left(k\left(z_{2}-z_{02}\right)\right)+C_{22} \sin \left(k\left(z_{2}-z_{02}\right)\right)+\frac{V_{02}}{2 j c} \sin \left(k\left|z_{2}-z_{02}\right|\right),
\end{aligned}
$$




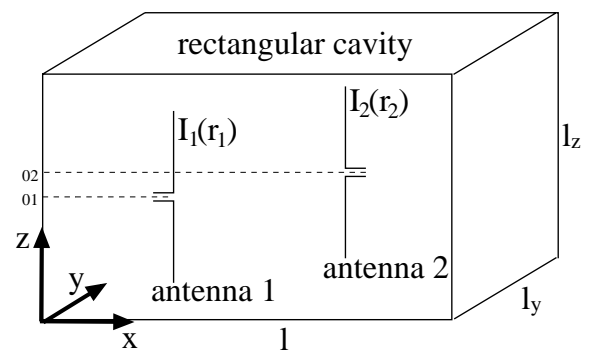

Fig. 2. Boundary value problem of two coupled antennas within a rectangular cavity. For a given excitation the currents $I_{1}$ and $I_{2}$ need to be determined.

where the integrals extend over antenna 1 and antenna 2 , as indicated. The voltages $V_{01}$ and $V_{02}$ are determined from the boundary conditions that are imposed at the antenna input terminals of antenna 1 and antenna 2, respectively. If a slice generator is present at an input terminal we have $V_{0}(t)=V_{0} \sin (\omega t)$, such that $V_{01}$ or $V_{02}$ assume constant values in this case. For a passive antenna with a load $Z_{L}$ the relation $V_{0}=-I\left(z_{0}\right) Z_{L}$ holds. This relation remains valid in the limiting cases $Z_{L} \rightarrow \infty$ (open circuited antenna) and $Z_{L} \rightarrow 0$ (short circuited antenna). The boundary conditions of vanishing current at the ends of the antennas will be enforced after discretization by means of the method of moments and determine the integration constants $C_{11}, C_{12}, C_{21}$, and $C_{22}$. In the method of moments procedure we will use pulse functions as basis functions and perform a point matching (Sadiku, 2000). To write a corresponding computer program is rather straightforward since, due to the Green's function approach, there is no need to discretize the cavity. The boundaries are already taken into account by the Green's function of the cavity.

In view of the calculation of the mutual impedance $Z_{12}$ we now turn to an instructive example. We consider a cavity with dimensions $l_{x}=6 \mathrm{~m}, l_{y}=7 \mathrm{~m}$, and $l_{z}=3 \mathrm{~m}$. These dimensions roughly correspond to the mode-stirred chamber at the University of Magdeburg. Within this cavity we place an active antenna 2 and an open-circuited antenna 1 . The coupled Hallén's Eqs. $(37,38)$ allow to calculate the antenna currents $I_{1}, I_{2}$ and to study the influence of the presence of the open-circuited antenna 1 onto the antenna current of antenna 2. Both antennas are taken of length $2 \mathrm{~m}$ and radius $10^{-3} \mathrm{~m}$. We focus on the mode $\nu=212$ with $n_{x}=2, n_{y}=1$, and $n_{z}=2$, compare Eq. (32). According to Eq. (31) this yields a wavenumber $k_{212} \approx 2.565 \mathrm{~m}^{-1}$ that corresponds to a frequency $f_{212} \approx 122.5 \mathrm{~Hz}$. With this frequency we excite antenna 2 by means of a slice generator of amplitude $V_{02}=1 \mathrm{~V}$. Moreover, for a mode-stirred chamber as resonator it is realistic to assume a quality factor $Q=1000$. A finite quality factor prevents infinities at resonance and is taken into account by a complex wavenumber according to $k \rightarrow k(1-j /(2 Q))$. We note that, in principle, the quality factor depends on the frequency and it is not possible to define an overall value. However, for our purposes it is sufficient to keep a constant

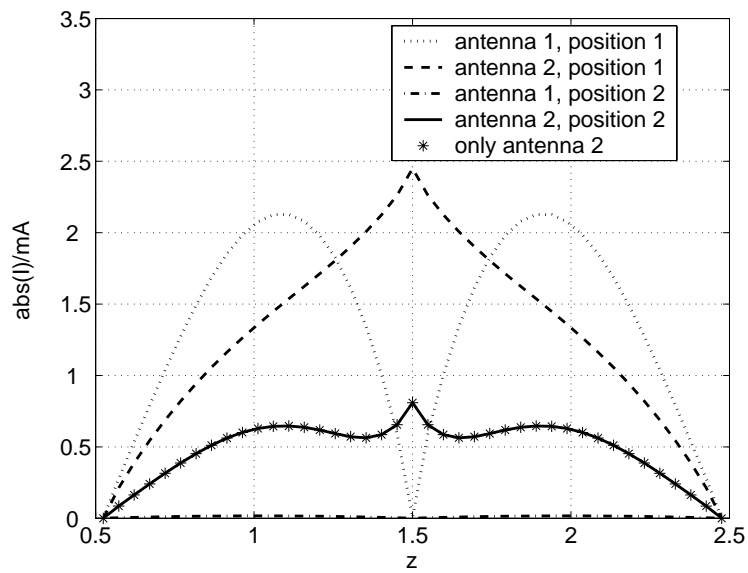

Fig. 3. Solution of Hallén's equation within a rectangular cavity in the coupled cases for position 1 and position 2 . The absolute values of the antenna currents as a function of position $z$ along each antenna are displayed. For comparison also the solution for the single antenna 2 is shown.

value of $Q$ since the determination of the quality factor is independent of the following calculation.

To begin with we align antenna 1 with the $z$-axis with its center at $z_{01}=1.5 \mathrm{~m}$ and put it at $x_{1}=1.5 \mathrm{~m}, y_{1}=3.5 \mathrm{~m}$. Also antenna 2 gets aligned to the $z$-axis with its center at $z_{02}=1.5 \mathrm{~m}$ but is put at $x_{2}=4.5 \mathrm{~m}, y_{1}=3.5 \mathrm{~m}$. This configuration will be referred to in the following as "position 1". In view of the general form of the eigenfunctions Eq. (32) it is clear that in position 1 both antennas will couple to the mode 212. The corresponding solution of the coupled Hallén's equations is shown in Fig. 3 where the absolute values of the antenna currents are displayed as dotted and dashed lines. It is obvious that on the open-circuited antenna 1 there is an induced current that is of comparable size in regard to the current of the active antenna 2. It follows, as we will also see from the next paragraph, that the presence of the opencircuited antenna 1 has a large influence on the active antenna 2.

As a second configuration we keep antenna 2 at its position and parallelly displace antenna 1 to $x_{1}=3 \mathrm{~m}, y_{1}=3.5 \mathrm{~m}$. This configuration will be referred to in the following as "position 2". The eigenfunction of the mode 212 vanishes at $x_{1}=3 \mathrm{~m}$, and this is why antenna 1 , in principle, will not couple to this mode in position 2. Now the solution of the coupled Hallén's equations yields the dashed-dotted and the solid line of Fig. 3. The induced current on the open-circuited antenna 1 is about three orders of magnitude smaller than the current on the active antenna 2. (It is not exactly zero due to the finite quality factor of the cavity which leads to a minor shift of the resonance and due to numerical inaccuracies.) Therefore at position 2 the presence of the open-circuited antenna 1 practically has no influence on antenna 2 . This is revealed if antenna 1 is completely removed and the current distribution on the single, active antenna 2 is calculated. This current distribution is represented in Fig. 3 by star-shaped 


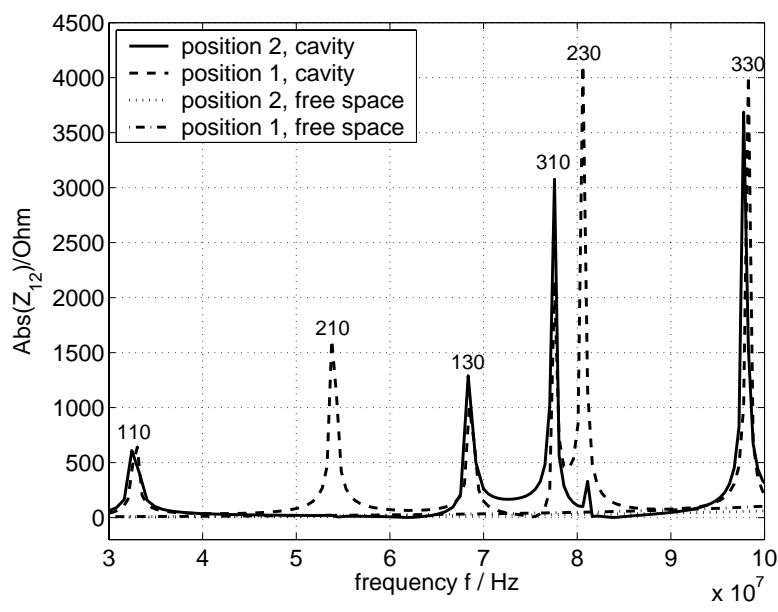

Fig. 4. Absolute value of the mutual impedance for position 1 and 2 in the frequency range of $30 \mathrm{MHz}$ to $100 \mathrm{MHz}$. The resonance peaks are labeled by the parameter $\nu=n_{x} n_{y} n_{z}$.

markers. The difference to the current distribution on antenna 2 for position 1 is obvious.

We finally turn to the computation of the mutual impedance $Z_{12}$ which is obtained from Eq. (23). Figure 4 shows the absolute value $\left|Z_{12}\right|$ between $30 \mathrm{MHz}$ and $100 \mathrm{MHz}$ for position 1 and 2. For comparison we also show for these positions the mutual impedance in absence of the cavity. The mutual impedance within the cavity is characterized by sharp resonance peaks that can be attributed to the various cavity modes and indicate the strong mutual coupling at resonance. It should be noted that due to the specific placement of the antennas not all cavity modes get excited. In Fig. 4 the modes $110(32.9 \mathrm{MHz}), 210(54.4 \mathrm{MHz})$, $130(69.0 \mathrm{MHz}), 310(78.0 \mathrm{MHz}), 230(81.4 \mathrm{MHz})$, and 330 $(98.8 \mathrm{MHz})$ can be identified. Modes with $n_{y}$ even or $n_{z}$ odd are not excited for positions 1 and 2. Moreover, in case of position 2 there is no excitation of modes with $n_{x}$ even. In between the different resonances the value of $\left|Z_{12}\right|$ within the cavity approaches that of free space.

In view of applications to EMC it becomes clear that the electromagnetic coupling between unintentionally coupled antennas within a cavity can be considerably reduced not only by avoiding resonance frequencies or decreasing the quality factor of the cavity. An equally important aspect is the positioning of the antennas within the cavity that should be chosen such that a simultaneous coupling to dominant modes is avoided. To do this in a systematic way requires a knowledge of the structure of the eigenmodes, of course.

\section{Conclusion}

The calculation of the mutual impedance between dipole antennas within a rectangular cavity is, in principle, straightforward. However, two steps deserve mentioning. One step is to check if the required integral expressions for the mutual impedance are also valid within a cavity, the second is to utilize a cavity's Green's function which is accurate and computationally efficient both in the source region and at resonance.

To solve a system of coupled integral equations that is based on Hallén's equation rather than on Pocklington's equation has numerical advantages. The integral kernels are less singular, leading to matrix systems within the methods of moments that are characterized by comparatively low condition numbers. To put this issue in the right perspective one should note that, quite generally, calculations of antenna impedances within resonating environments of high quality factors and near resonance a priori are plagued by numerical instabilities. In this context the Green's function approach, if applied by means of a computationally efficient series representation as above, proves to be advantageous.

It should be admitted that we have investigated a generic example. The cavity's Green's function approach clearly fails if the cavity under investigation is not of canonical shape such that its Green's function is not known. Also the coupled system of Hallén's equation becomes more complex if more complicated antenna configurations are under consideration. Especially the determination of the incident vector potential and its associated integration constants becomes cumbersome if more antennas with arbitrary orientation have to be investigated.

Finally we stress again that within a cavity the mutual impedance between two dipole antennas critically depends on their absolute position within the cavity. This is in contrast to free space where the relative position of the antennas is decisive. In particular, within a cavity the mutual coupling does not necessarily decay in case of increasing distance between the two antennas since the cavity modes do not decay with increasing distance, as well. For strong antenna coupling at resonance it is not the relative distance but the simultaneous coupling to a resonant mode that is important.

\section{References}

Elliott, R. S.: Antenna theory and design, Prentice-Hall, Englewood Cliffs, 1981.

Ewald, P. P.: Die Berechnung optischer und elektrostatischer Gitterpotentiale, Annalen der Physik vol. 64, 253-268, 1921.

Gronwald, F., Nitsch, J. and Tkachenko, S: Hybrid representation methods for the efficient analysis of antenna coupling within cavities, EMC 2002-Proc.of the Sixteenth International Wroclaw Symposium on Electromagnetic Compatibility, Wroclaw, Poland, June 2002, 109-114, 2002.

Gronwald, F.: The influence of electromagnetic singularities on an active dipole antenna within a cavity, Advances in Radio Science, vol. 1, 57-61, 2003.

Jordan, K. E., Richter, G. R., and Sheng, P.: An Efficient Numerical Evaluation of the Green's Function for the Helmholtz Operator on Periodic Structures, Journal of Computational Physics, vol. 63, 222-235, 1986.

Lee, K. S. H. (Ed.): EMP Interaction: Principles, Techniques, and Reference Data, revised printing, Taylor \& Francis, Washington D.C., 1995. 
Nakano, H.: Antenna Analysis using Integral Equations, in: Analysis Methods for Electromagnetic Wave Problems, Volume Two, edited by: Yamashita, E., Artech House, Boston, 1996.

Park, M.-J., Park, J., and Nam, S.: Efficient Calculation of the Green's Function for the Rectangular Cavity”, IEEE Microwave and Guided Wave Letters, vol. 8, 124-126, March 1998.

Sadiku, M. N. O.: Numerical Techniques in Electromagnetics, 2nd. Ed., CRC Press, Boca Raton, 2000.
Senior, T. B. A. and Volakis, J. L.: Approximate boundary conditions in electromagnetics, (IEE, London, UK), 1995.

Tai, C.-T.: Dyadic Green Functions in Electromagnetic Theory, IEEE Press, New York, 1994.

Tesche, F. M., Ianoz, M. V., and Karlsson, T.: EMC Analysis Methods and Computational Methods, John Wiley \& Sons, New York, 1997.

Van Bladel, J.: Electromagnetic Fields, revised printing, Hemisphere Publishing, New York, 1985. 